\title{
Editorial
}

Nephrology

\section{Recognizing Downstream Consequences of Acute Kidney Injury}

\author{
Lenar Yessayan Michael Heung \\ Department of Medicine, Division of Nephrology, University of Michigan, Ann Arbor, MI, USA
}

Acute kidney injury (AKI) complicates up to $20 \%$ of hospitalizations and is associated with poor short-term outcomes including prolonged hospital stay, high risk of hospital readmission, and high short-term mortality [1]. In recent years, a variety of epidemiological studies have also shown that survivors of AKI remain at risk for subsequent complications, including recurrent AKI, progressive kidney disease, elevated blood pressure, cardiovascular events, and long-term mortality.

In this issue of the American Journal of Nephrology, Lee et al. [2] contribute further to our understanding of the lingering, long-term impact of AKI. Using the USRDS database with linked Medicare part A and B claims, the authors identified 47,341 patients initiated on in-center hemodialysis and examined whether AKI events in the 2 years prior to end-stage renal disease (ESRD) diagnosis impacted outcomes, specifically the odds of starting dialysis with a functioning arteriovenous access and allcause mortality. Patients were followed from dialysis initiation for up to 3 years. One-year all-cause mortality was considerably higher in patients with pre-ESRD AKI compared to those without these events ( 38 vs. $25 \%$ respectively, $p<0.001)$. After adjusting for several clinically important covariates such as cardiovascular disease, cardiovascular and infection-related hospitalization, and pre-ESRD nephrology care, the odds of starting hemodialysis with arteriovenous access was significantly lower in

\section{KARGER}

(c) 2018 S. Karger AG, Basel

E-Mail karger@karger.com

www.karger.com/ajn patients with pre-ESRD AKI event versus those without pre-ESRD AKI event (OR 0.47; 95\% CI 0.44-0.50). In another adjusted model with similar covariates that also included type of dialysis access, the odds of all-cause mortality was significantly higher in patients with pre-ESRD AKI event compared to those without pre-ESRD AKI event (OR 1.36; 95\% CI 1.30-1.42).

Strikingly, over half (54\%) of the ESRD patients in this study suffered an episode of AKI in the 2 years preceding ESRD. This finding is even more remarkable when considering that AKI was defined using administrative data which tends to identify more severe cases and underestimate the overall incidence of AKI. Presumably many of these patients already had chronic kidney disease (CKD) as a predisposing risk factor for AKI. In turn, the AKI may have accelerated CKD progression toward ESRD, or contributed to de novo CKD in other cases. Regardless, this finding highlights the bidirectional relationship between $\mathrm{AKI}$ and CKD, and emphasizes the critical role of AKI in the progression or development of CKD, either as a cause or consequence.

How do we explain the independent association between pre-ESRD AKI event(s) and lower likelihood of permanent arteriovenous access at hemodialysis initiation? In the multivariable model, Lee et al. [2] adjusted for several potentially confounding factors, but a couple of additional factors bear further mention not only for 
statistical purposes but as potential opportunities to intervene. First, peripherally inserted central catheters (PICCs) are increasingly used in hospitalized patients for medication administration, multiple laboratory draws, and total parenteral nutrition. Even short-term use of PICC lines has been associated with thrombosis, stenosis, and obliteration of central and peripheral veins. These complications may lead to premature exhaustion of veins available for future fistula construction. Indeed, McGill et al. [3] have reported that PICC exposure in the 2 years preceding dialysis initiation is independently associated with lower likelihood of transition to any working fistula or graft. Second, the duration between AKI episode and ESRD will in part define whether there is adequate time to place an arteriovenous access. Lee et al. [2] found that $50 \%$ of the patients with a pre-ESRD AKI event had their last AKI hospitalization within 1 month of ESRD initiation, which would not have allowed for vascular access placement and maturation. Whether that subset of patients had preceding AKI events or could have otherwise been identified as being at risk for progression to ESRD may be worth exploring in a future study.

It is well recognized that the ESRD state confers excess mortality when compared to the general population. Death due to cardiovascular and non-cardiovascular causes in the ESRD population far exceeds that in the non-ESRD population [4]. A recent meta-analysis suggested an independent association between AKI and long-term cardiovascular disease including congestive heart failure, stroke, and acute myocardial infarction [5]. Lee et al. [2] further contributed to this link by describing the association between AKI events 2 years preceding ESRD and increased risk of 1-year mortality in a group of patients with an already high risk for cardiovascular and overall mortality. This effect appears to be independent of the study's other key finding regarding vascular access, as access type was accounted for in the adjusted models. Exposure to an episode of AKI could be associated with pathophysiologic changes, such as inflammation and vascular stress, which could contribute to cardiovascular disease. Conversely, the predisposing factors leading to AKI could serve as unmeasured confounders for cardiac outcomes. Regardless, clinicians must be aware of the increased risk of adverse outcomes in patients with a history of AKI and actively identify such patients. Of note, the current ESRD medical evidence report form (CMS form 2728) does not include AKI as a comorbid condition, and AKI is often not listed as the primary cause of ESRD when other contributing factors are present.
As evidence continues to mount of the deleterious downstream consequences of AKI, more emphasis needs to be placed on prevention. Primary prevention is no longer just a theoretical concept, as recent studies have demonstrated the ability to either prevent some cases of AKI altogether or attenuate the severity of AKI [6]. These efforts have succeeded not through the use of novel therapies, but rather with a focus on identifying high-risk patients and applying basic nephro-protective approaches, such as avoidance of nephrotoxins, close monitoring of kidney function, and hemodynamic optimization.

Beyond primary prevention, there is also significant opportunity to mitigate the secondary consequences of AKI. As discussed earlier, the use of PICC lines in such patients may have serious negative downstream consequences regarding dialysis vascular access, and should be avoided whenever possible (and certainly in patients with pre-existing CKD). Nephrology follow-up must be prioritized, at least in high-risk cases, to ensure monitoring of kidney function and avoidance of unnecessary nephrotoxic exposures. More research is needed to identify measures (pharmacologic or otherwise) to enhance kidney function recovery or slow down CKD development/progression after an episode of AKI. However, even in the absence of such therapeutic options, nephrologists can provide appropriate CKD/ESRD education and planning; in at least one study, nephrology care after severe AKI has been associated with reduced mortality [7], yet post-AKI nephrology rates remain low overall.

Taken together, the association between AKI events, substantial 1-year mortality after onset of ESRD, and the lower likelihood of a working fistula or graft should receive more attention from clinicians. AKI cannot be thought of solely as an acute complication, even in cases of recovery, but rather needs to be viewed as a major risk factor for long-term adverse outcomes, including after ESRD initiation. Unfortunately, there remain large gaps in the care of patients with AKI including failure of recognition, suboptimal management, and lack of follow-up [8]. In this era of high-value care, health systems should be dedicating significant resources to quality improvement programs for AKI risk reduction as well as secondary prevention of downstream complications. Nephrologists would do well to lead those efforts.

\section{Disclosure Statement}

Neither author has any relevant conflicts of interest or financial disclosures. 


\section{References}

1 Susantitaphong P, Cruz DN, Cerda J, et al: World incidence of AKI: a meta-analysis. Clin J Am Soc Nephrol 2013;8:1482-1493.

$\longrightarrow 2$ Lee T, Shah S, Leonard AC, Parikh P, Thakar CV: Acute kidney injury before dialysis initiation predicts adverse outcomes in hemodialysis patients. Am J Nephrol 2018;47:427-434.

- 3 McGill RL, Ruthazer R, Meyer KB, Miskulin DC, Weiner DE: Peripherally inserted central catheters and hemodialysis outcomes. Clin J Am Soc Nephrol 2016;11:1434-1440. de Jager DJ, Grootendorst DC, Jager KJ, et al: Cardiovascular and noncardiovascular mortality among patients starting dialysis. JAMA 2009;302:1782-1789.

Odutayo A, Wong CX, Farkouh M, et al: AKI and long-term risk for cardiovascular events and mortality. J Am Soc Nephrol 2017;28: 377-387.

Meersch M, Schmidt C, Hoffmeier A, et al: Prevention of cardiac surgery-associated AKI by implementing the KDIGO guidelines in high risk patients identified by biomarkers: the PrevAKI randomized controlled trial. Intensive Care Med 2017;43:1551-1561.

7 Harel Z, Wald R, Bargman JM, et al: Nephrologist follow-up improves all-cause mortality of severe acute kidney injury survivors. Kidney Int 2013;83:901-908.

-8 Aitken E, Carruthers C, Gall L, Kerr L, Geddes C, Kingsmore D: Acute kidney injury: outcomes and quality of care. QJM 2013;106: 323-332. 